\title{
SARS-CoV-2 and pediatric encephalopathy: Google analytics and predictive modeling
}

\author{
Hend Jaddoa Jassim ${ }^{1,2,3}$, Shaima Hussein Alwan ${ }^{1,2}$, Ahmed Al-Imam $^{4,5}$, \\ Huda Adnan Hussein ${ }^{6}$, Ali Kamil Al-Shalchy ${ }^{7,8,9}$ \\ ${ }^{1}$ Specialist Pediatrician, Department of Pediatrics, Diyala Health Directorate, Iraqi Ministry of Health, ${ }^{2}$ Fellow, Department \\ of Pediatrics, The Iraqi Board for Medical Specialties, Iraq, ${ }^{3}$ Member, Department of Pediatrics. The Council of Arab \\ Board of Health Specializations, Syria, ${ }^{4}$ Senior Researcher, Department of Anatomy and Cellular Biology, College of \\ Medicine, University of Baghdad, Iraq, ${ }^{5}$ Associate Researcher, Department of Computer Science and Statistics, PUMS \\ Doctoral School, Poznan University of Medical Sciences, Poland, ${ }^{6}$ Resident Doctor, Department of Pediatrics, Diyala \\ Health Directorate, Iraqi Ministry of Health, ${ }^{7}$ Dean of the College of Medicine, University of Baghdad, Iraq, ${ }^{8}$ Consultant \\ Neurosurgeon, Department of Surgery, Neurosurgical Unit, College of Medicine, University of Baghdad, Baghdad, Iraq, \\ ${ }^{9}$ Fellow of the Royal Colleges of Surgeons, United Kingdom
}

A B S TR A C T

Background: The severe acute respiratory syndrome coronavirus 2 (SARS-CoV-2) infection is rare in children and possesses distinctive clinical features. Aims and Objectives: The primary objective is to bring clinicians,' researchers,' and epidemiologists' attention to pediatric encephalopathy as part of the clinical manifestations of SARS-CoV-2 in children. Materials and Methods: Google analytics aimed to explore the spatial-temporal (geographicchronological) mapping of SARS-CoV-2 in conjunction with pediatric encephalopathy and encephalitis. We retrieved longitudinal data from Google Trends, for one year starting from December 2019, by deploying five search topics; "SARS-CoV-2," "Coronavirus disease 2019, COVID-19" "Pediatric Encephalopathy," "Pediatric Encephalitis," and "Encephalitis in Children." Results: Spatio-temporal mapping was most conclusive for "COVID-19" and "Severe acute respiratory syndrome coronavirus 2." Internet users were least interested in topics related to pediatric encephalopathy and encephalitis in children; potentially, reflecting the rarity of these entities in SARS-CoV-2 infections in children. We are also reporting a case of atypical SARS-CoV-2 in an 8-year-old child, in which pediatric encephalopathy occurred in a PCR-confirmed COVID-19 case. Conclusion: Google analytics reconciled with the case report. SARS-CoV-2 in children may present with neurological rather than respiratory manifestations, which is atypical and rare. We are conveying two key messages; (1) pediatricians can collaborate with data scientists to realize evidence-based pediatric medicine and (2) digital data are worthy of exploration to guide subsequent rigor research, including randomized controlled trials and meta-analytics.

Key words: COVID-19; Internet; Pediatric encephalitis; Pediatric encephalopathy; SARS-COV-2; Surface web

\section{INTRODUCTION}

During the past two decades, coronaviruses caused two epidemics, the severe acute respiratory syndrome (SARS) and the Middle East Respiratory Syndrome in 2002-2003 and 2012, respectively. ${ }^{1}$ The severe acute respiratory syndrome coronavirus-2 (SARS-CoV-2), which is responsible for the coronavirus disease 2019 (COVID-19), is a virulent pathogenic zoonotic viral infection. ${ }^{2}$ By the end of 2019, COVID-19 spread exponentially in the

Address for Correspondence:

Dr. Ahmed Al-Imam, Senior Researcher, Department of Anatomy and Cellular Biology, College of Medicine, University of Baghdad, Iraq.

Phone: +964 (0) 7714338199 [Iraq], +1 (581) 700-0110 [Canada]. E-mail: tesla1452@gmail.com,

ahmed.mohammed@comed.uobaghdad.edu.iq 
city of Wuhan in China, in which the Chinese Centre for Disease Control and Prevention reported numerous ambiguous cases of pneumonia of an unknown etiology among the Wuhan residents; microbiologists identified a novel coronavirus based on clinical samples and genomic analysis. ${ }^{1,2}$

Since the initial reports of the Wuhan outbreak, the SARS$\mathrm{CoV}-2$ pandemic has been spreading around the world at an alarmingly exponential rate. ${ }^{3}$ As August 13, 2021, the number of confirmed infections exceeded 206,457,258 worldwide and 1,761,143 in Iraq; complications related to the illness claimed the lives of over 4,352,521 globally and 19,541 individuals in Iraq. ${ }^{3}$ SARS-CoV-2 affected nations from the developed and the developing world; the top ten countries included the United States, India, Brazil, Russia, France, United Kingdom, Turkey, Argentina, Columbia, and Spain. ${ }^{3}$

As a top priority for the global health agenda, scientists successfully developed several effective vaccines, including Pfizer's and Moderna's, with the proclaimed $95 \%$ and $94.5 \%$ effectiveness, respectively. ${ }^{4,5}$ However, novel mutant strains of the COVID-19 emerged, including the recently identified delta $(\boldsymbol{\delta})$ variant in India that infected nearly 31 million individuals and killed over 430,000 Indians. $3,6,7$

\section{Aims and objectives}

Our study comprises an amalgam of a case report and collateral analytics of online information search behavior retrieved through the Google Trends database from 2019 to 2020. The primary objective is to bring readers' attention, including clinicians, researchers, and epidemiologists, to the potential association of pediatric encephalopathy (or encephalitis) with the clinical manifestations in children infected with SARS-CoV-2. Concerning the secondary objective, Google search engine analytics will attempt to highlight the potential deficit of knowledge of web users, including patients and physicians, concerning the primary objective, by scrutinizing the spatio-temporal mapping of the web search volume using specific search topics. Thereby, we highlight the noteworthiness of modalities of frequentist (non-Bayesian) statistics and predictive analytics to the Iraqi authorities, specifically the Iraqi Ministry of Health.

\section{MATERIALS AND METHODS}

The authors carried out the work described in this manuscript following the code of ethics of the Declaration of Helsinki on medical research, the ethical principles defined in the Farmington Consensus, and the uniform requirements for manuscripts submitted to biomedical journals. The ethics committee of the College of Medicine at the University of Baghdad approved the study protocol.

We retrieved raw data through Microsoft Google Trends engine and conducted data analyses using IBM-SPSS version 24 and Microsoft Office Excel 2016 with Analysis ToolPak add-in.

Google Analytics aimed to explore the spatial (geographic) and temporal (chronological) mapping of SARS-CoV-2 in conjunction with pediatric encephalopathy and encephalitis. Spatial-temporal mapping relies on web search volume reflecting the interest of internet (surface web) users concerning specific web queries. We retrieved longitudinal data from Google Trends, for the period from December 1, 2019, to December 1, 2020, that is, during the pandemic, using five search topics; "SARS-CoV-2," "COVID-19," "Pediatric Encephalopathy," "Pediatric Encephalitis," and "Encephalitis in Children."

\section{RESULTS}

\section{Google trends analytics}

Principally, spatial mapping conveyed data concerning the first two search topics; SARS-COV-2 and COVID-19. Data signals originated from 46 countries; the first twenty of which included Canada, New Zealand, Portugal, Ireland, South Africa, France, Chile, India, Australia, United States, Peru, United Kingdom, Mexico, Ecuador, Romania, Colombia, Indonesia, Spain, Thailand, and Brazil (Figure 1). Only six nations (13.04\%) from the Arab world and the Middle East contributed to the holistic map, including Iran, Turkey, Morocco, United Arab Emirates, Saudi Arabia, and Egypt. Concerning the other three search topics; "Pediatric Encephalopathy," "Pediatric Encephalitis," and "Encephalitis in Children," geographic

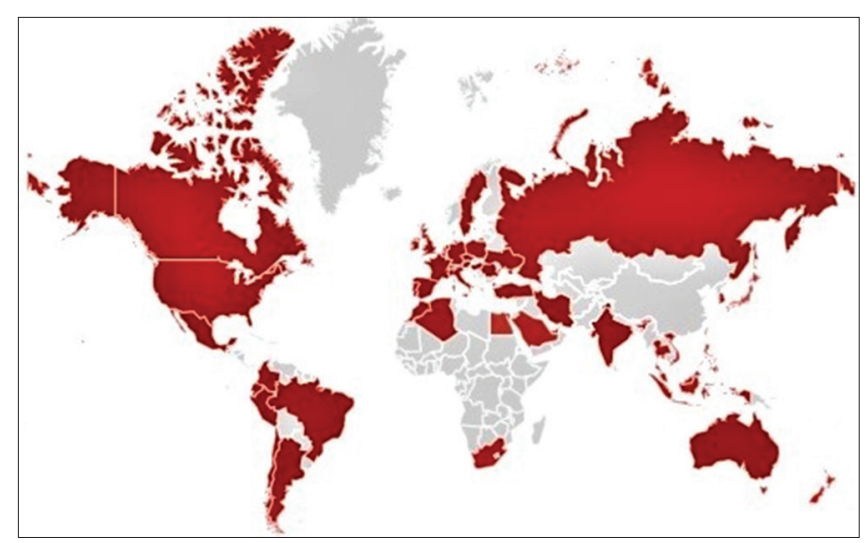

Figure 1: Spatial mapping of web queries. *Date: $01 / 12 / 2019$ to $01 / 12 / 2020$ 
mapping attained the highest levels in the United States, India, and South Africa, respectively. Web-users from the Arab world and the Middle East did not display interest in any of those topics.

Concerning the chronological mapping, statistical outliers existed during March and April 2020; these were related to SARS-CoV-2 and COVID-19 search topics only. Besides, internet users did not show interest in these topics before January 2020. Web queries exhibited high temporal variability (Figure 2), for the period from December 1, 2019, to December 1, 2020, for SARS-CoV-2 (mean $[ \pm$ standard error $]=0.98[ \pm 0.166]$, skewness $=2.289$, and kurtosis $=4.893)$ and COVID-19 (22.18 [ \pm 2.885$]$, $0.861,-0.684)$. Predictive modeling (Table 1) succeeded in generating equations of the sixth order of polynomial function for SARS-CoV-2 $\left(\mathrm{R}^{2}\right.$ score $=0.7384$, correlation coefficient $=0.8593)$ and COVID-19 $\left(\mathrm{R}^{2}\right.$ score $=0.7600$, correlation coefficient $=0.8718)$; each has a strong effect size. Predictive modeling was not successful for the other search topics.

Significant bivariate correlations existed only for SARSCoV-2 versus COVID-19 (Kendall's tau-b $[\tau \mathrm{b}]=0.788$, $P<0.001)$; correlation was strongly positive. Further, we compared the web search volume across all search topics using nonparametric statistics. Friedman test confirmed a statistically significant difference (Chi-square $=181.689$, df $=4, P<0.001$ ), while post hoc analysis using Wilcoxon signed-rank test validated a significant difference for SARSCoV-2 versus COVID-19 (standardized test statistic = $5.743, P<0.001)$, and in favor of the second search topic (COVID-19).

To summarize, spatial and temporal mapping were most conclusive for two search topics only; "COVID-19" and "SARS-CoV-2." Hence, predictive models were not feasible for the other topics. Further, inferential data on web search

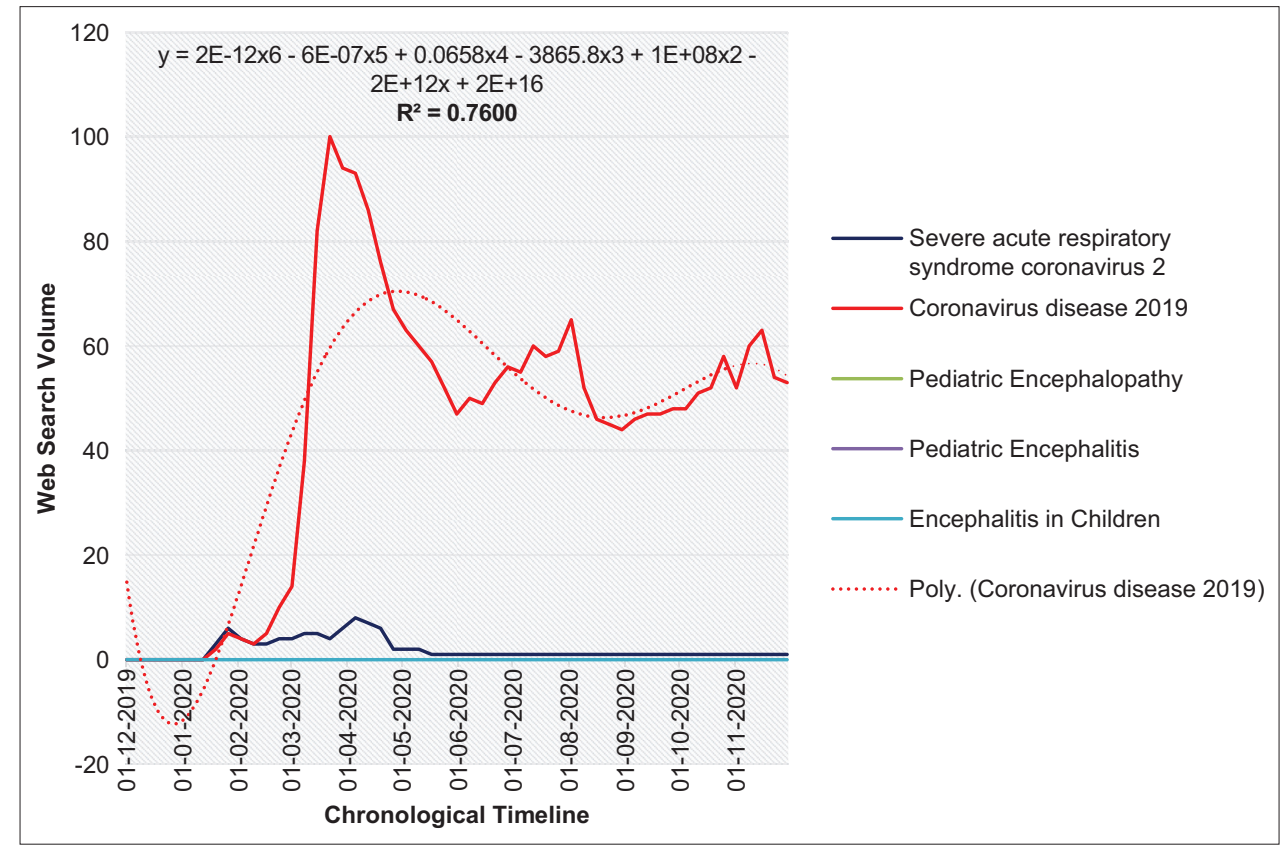

Figure 2: Temporal mapping of the web search volume. ${ }^{\star}$ Date: $01 / 12 / 2019$ to $01 / 12 / 2020$

\begin{tabular}{|c|c|c|c|c|c|}
\hline Search topic & Equation of the predictive model & $\begin{array}{l}\text { Order of polynomial } \\
\text { function }\end{array}$ & $r^{2}$ score & $\begin{array}{l}\text { Correlation } \\
\text { coefficient }\end{array}$ & $\begin{array}{l}\text { Strength of } \\
\text { correlation }\end{array}$ \\
\hline $\begin{array}{l}\text { "Severe acute respiratory } \\
\text { syndrome coronavirus } 2 \text { " }\end{array}$ & $\begin{array}{l}y=8 E-08 x^{6}-1 E-05 x^{5}+0.001 x^{4}- \\
0.0296 x^{3}+0.4003 x^{2}-1.6935 x+1.7466\end{array}$ & $6^{\text {th }}$ & 0.7384 & 0.8593 & Strong \\
\hline $\begin{array}{l}\text { "Coronavirus disease } \\
\text { 2019" }\end{array}$ & $\begin{array}{l}y=3 E-07 x^{6}-6 E-05 x^{5}+0.0051 x^{4}- \\
0.2053 x^{3}+3.7559 x^{2}-23.68 x+34.947\end{array}$ & $6^{\text {th }}$ & 0.7600 & 0.8718 & Strong \\
\hline "Pediatric Encephalopathy" & N/A & N/A & N/A & $\mathrm{N} / \mathrm{A}$ & N/A \\
\hline "Pediatric Encephalitis" & N/A & N/A & N/A & $\mathrm{N} / \mathrm{A}$ & $\mathrm{N} / \mathrm{A}$ \\
\hline "Encephalitis in Children" & $\mathrm{N} / \mathrm{A}$ & N/A & $\mathrm{N} / \mathrm{A}$ & N/A & $\mathrm{N} / \mathrm{A}$ \\
\hline
\end{tabular}


queries verified that internet users were least interested in topics related to pediatric encephalopathy and encephalitis; potentially, reflecting the rarity of these manifestations as part of the clinical picture of SARS-CoV-2 infections in children.

\section{Case report}

A 6-year-old male child, by the pseudonym "Muhammed," from Ba'quba in Diyala governorate; the chief complaint was abnormal body movement, identified as seizures, for which the parents admitted him to the emergency unit at Al-Batool Teaching Hospital. He is a known case of type-1 diabetes mellitus and receiving insulin therapy. His medical condition began 10 days earlier; he had an elevated core body temperature (pyrexia) uncontrolled by antipyretics; 2 days before hospital admission, he had abdominal pain and arthritic knee pain unresponsive to analgesia. On the same day of admission to the hospital, he developed abnormal body movement, and his family brought him to the emergency room (ER). In the ER, the patient was drowsy; his random blood sugar (RBS) was elevated $(369 \mathrm{mg} / \mathrm{dl})$. Systemic review confirmed that the patient had no cyanosis, no edema, no cough, no hemoptysis, no hematuria, no dysuria, and no skin rash. The patient gradually became unconscious due to status epilepticus.

The mother had a normal prenatal history; she was healthy during pregnancy, and she did not develop diabetes mellitus, hypertension, or any other ailment. Regarding natal history, the child was the product of a normal vaginal delivery at home; there were no complications, and he cried immediately following birth, and he had no resuscitation. Concerning the medical and surgical history, he had one seizure attack 1 year earlier due to hypoglycemia, and he had a circumcision by the age of 1 year. Developmental history was entirely normal; he began to smile at the age of 1 month; at the age of 8 months, he could sit without any support; at the age of 10 months, the child started to say "mama" and "baba;" at the age of 13 months, he was able to walk on his own. Regarding the family history, Muhammed's uncle also had type- 1 diabetes mellitus; the family never had a history of epilepsy. The child had no history of allergy to any medication.

On physical examination, the heart rate was 112 beats per minute, a blood pressure of $98 / 60 \mathrm{mmHg}$, a temperature of 37.4 Celsius, and a respiratory rate of 22 breathes per minute. Concerning laboratory examination, a complete blood picture confirmed that the child had lymphocytosis $(67.5 \%)$, thrombocytopenia $\left(122 * 10^{9} / \mathrm{L}\right)$, and positive CRP. Serum electrolytes were entirely normal except for elevated serum magnesium $(2.6 \mathrm{mg} / \mathrm{dl})$. Urine examination indicated the presence of ascorbic acid (+) and glucose
$(++)$. Further, chest X-ray was normal, and rapid COVID-19 testing showed negative IgG and IgM.

At the ER, the attending physicians initiated diabetic ketoacidosis regime; several hours later, the patient had normal RBS readings. Nonetheless, the patient had recurrent fits, generalized tonic-clonic seizures, although receiving anticonvulsant therapy. Therefore, the clinicians admitted him to the intensive care unit (ICU), where the fits persisted despite having normal blood sugar. Later, he developed apnea; the intensive care specialist decided to conduct endotracheal intubation. On examination, the child was unconscious, had a clear chest, and had a good air entry.

Further, he had a soft abdomen, no organomegaly, no edema, the pupils were equal in size and reactive to light, and he had a positive gag reflex. The intensive care physician managed the patient with phenobarbital and phenytoin; however, there was no improvement clinically. Accordingly, the intensive care physician managed the child using midazolam and propofol, but the seizures never stopped. Muhammed was sent for radiology examination; computerized tomography (CT) of the brain was thoroughly normal; however, the radiologist communicated that an infection could not be excluded, mandating correlating the clinical findings with laboratory data. Besides, Muhammed also had a persistent fever in the ICU despite having antibiotic therapy. Accordingly, the specialist pediatrician had a clinical suspicion of infection, including urinary tract infection, ventilator-associated pneumonia, phlebitis due to a contaminated venous line, and an atypical SARS-CoV-2 infection. Therefore, the pediatrician sent Muhammed for comprehensive laboratory investigations, including a PCR test for COVID-19 through a nasal swab, which was positive. Subsequently, Muhammed was admitted to the quarantine unit. Unfortunately, the patient's condition continued to deteriorate, and he eventually died. Based on the clinical and laboratory findings, the managing physicians postulated a hypothesis for the differential diagnosis, including meningitis, encephalitis, or meningoencephalitis in association with an atypical SARS-CoV-2 infection.

\section{DISCUSSION}

In adults, COVID-19 manifests as fever and other constitutional clinical features, including chills, fatigue, and muscle aches. ${ }^{910}$ Later, the patient develops dry cough and dyspnea, while rhinitis and sore throat are rare, and some of them may develop diarrhea later., ${ }^{910}$ During the early phase of COVID-19, some individuals may develop a loss of smell and taste. ${ }^{2}$ Initially in Wuhan, almost one-third of patients required intensive care hospitalization, and relatively a high mortality $(10 \%)$, while higher mortality 
rates $(50 \%)$ existed in patients over the age of 50 years, in whom death usually occurs during the $3^{\text {rd }}$ week from the onset of symptoms. ${ }^{2}$ SARS-CoV-2 manifestations were more severe in elderly patients and those with pre-existing medical and surgical conditions, including cardiovascular and immunological disturbances. ${ }^{2}$

Concerning the opposite end of the age spectrum, clinical manifestations are obscure in children, while epidemiological data are still lacking concerning its exact prevalence. According to a systematic review by De Souza et al., (2020), who analyzed 38 studies (1124 cases), confirmed that clinical manifestations in children with COVID-19 differ drastically from adult cases; fever and respiratory symptoms should not be considered a hallmark of COVID-19 in children. ${ }^{11}$ De Souza and collaborators categorized COVID-19 cases based on their severity; $14.2 \%$ were asymptomatic, $36.3 \%$ were mild, $46.0 \%$ were moderate, $2.1 \%$ were severe, and $1.2 \%$ were critical. ${ }^{11}$ The most prevalent symptoms were fever $(47.5 \%)$, cough (41.5\%), nasal symptoms $(11.2 \%)$, diarrhea $(8.1 \%)$, and nausea/vomiting (7.1\%). More than one-third of children $(36.9 \%)$ were diagnosed with pneumonia, and one-tenth $(10.9 \%)$ had upper respiratory tract infections, and only one incident of death occurred. ${ }^{11}$

We recommend clinical researchers to incorporate online information search behavior and digital knowledge to complement classical epidemiological and experimental approaches concerning pediatric encephalopathy and encephalitis in children with SARS-CoV-2 infections. We urge continuous medical development professionals to boost the awareness of health-care practitioners, including pediatricians and pediatric neurologists, concerning the possibility of encountering pediatric encephalopathy in association with COVID-19 infection. We also prompt research methodologists and data scientists to attempt conforming non-Bayesian statistics with machine learning models to explore this particular health problem. Therefore, we highlight key messages: (1) Pediatricians can collaborate with data scientists to realize the importance of big data for evidence-based pediatric medicine by investing in real-time analytics, predictive models, and machine learning. ${ }^{12-16}$ (2) Digital data are worthy of exploration from an epidemiological perspective to guide subsequent research of the higher level-of-evidence hierarchy, including randomized controlled trials and meta-analytic studies. ${ }^{17}(3)$ Our research methodology and results are novel and valuable for subsequent aggregate studies.

\section{Limitations of the study}

Observational studies have a lower level of evidence than experimental studies, and they are more prone to bias and confounding variables. Besides, they may not be used to demonstrate causality. Therefore, we recommend that researchers explore experimental and quasi-experimental studies or combine these with observational studies to boost the level of evidence. Another way to overcome limitations is to consider digital epidemiological knowledge in conjunction with observational studies and deploy robust data analytics by reconciling non-Bayesian (Frequentist) statistics and machine learning techniques.

\section{CONCLUSIONS}

Spatio-temporal mapping was most conclusive for "COVID-19" and "SARS-CoV-2" predictive models were not feasible for the other topics. Further, inferential data on web search queries confirmed that surface web users were least interested in topics related to pediatric encephalopathy and encephalitis in children; potentially, reflecting the rarity of these entities in association with SARS-CoV-2 infections in children. Forty-six countries contributed to the holistic geographic map of online search behavior; almost one-eighth was from the Arab world and the Middle East, including Iran, Turkey, Morocco, United Arab Emirates, Saudi Arabia, and Egypt. Concerning the other three search topics; "Pediatric Encephalopathy," "Pediatric Encephalitis," and "Encephalitis in Children," spatial mapping attained the highest levels in the United States, India, and South Africa, respectively, while webusers from the Arab world and the Middle East did not display interest in any of those topics.

\section{AVAILABILITY OF DATA}

Our data, including the raw dataset, are available on request from the corresponding author.

\section{REFERENCES}

1. Al-Imam A, Khalid $U$ and Al-Doori HJ. Clustering analysis of coronavirus disease 2019 pandemic. Asian J Med Sci. 2021;12(2):108-113.

https://doi.org/10.3126/ajms.v12i2.33401

2. Motyka MA, Al-Imam A and Aljarshawi MH. SARS-CoV-2 pandemic as an anomie. Przestrzeń Społeczna (Social Space). 2020;20(2):111-144.

3. Worldometer. COVID-19 Coronavirus Pandemic. Available from: https://www.worldometers.info/coronavirus [Last accessed on 2021 May 03].

4. Livingston EH. Necessity of 2 doses of the Pfizer and Moderna COVID-19 vaccines. J Am Med Assoc. 2021;325(9):898. https://doi.org/10.1001/jama.2021.1375

5. Meo SA, Bukhari IA, Akram J, Meo AS and Klonoff DC. COVID-19 vaccines: Comparison of biological, pharmacological characteristics and adverse effects of Pfizer/BioNTech and moderna vaccines. Eur Rev Med Pharmacol Sci. 2021;25(3):1663-1669.

https://doi.org/10.26355/eurrev_202102_24877 
6. BBC News. "Double Mutant": What are the Risks of India's New Covid-19 Variant. Available from: https://www.bbc.com/news/ world-asia-india-56517495 [Last accessed on 2021 May 03].

7. MedPage Today. India's COVID-19 Variant: What We Know So Far. Available from: https://www.bbc.com/news/world-asiaindia-56517495 [Last accessed on 2021 May 03].

8. Nuti SV, Wayda B, Ranasinghe I, Wang S, Dreyer RP, Chen SI, et al. The use of google trends in health care research: A systematic review. PLoS One. 2014;9(10):e109583. https://doi.org/10.1371/journal.pone.0109583

9. Al-Imam A, Khalid U and Al-Doori HJ. Predictive epidemiology for SARS-CoV-2 pandemic in Iraq. Asian J Med Sci. 2021;12(3): 121-124.

https://doi.org/10.3126/ajms.v12i3.33402

10. Al-Imam A, Motyka MA and Al-Doori HJ. Surface web merits for SARS-CoV-2 pandemic in Iraq. J Facult Med Baghdad. 2020;62(4):117-127. https://doi.org/10.32007/jfacmedbagdad.6241795

11. De Souza TH, Nadal JA, Nogueira RJ, Pereira RM and Brandão MB. Clinical manifestations of children with COVID-19: A systematic review. Pediatr Pulmonol. 2020;55(8):1892-1899. https://doi.org/10.1002/ppul.24885

12. Al-Imam A and Al-Lami F. Machine learning for potent dermatology research and practice. J Dermatol Dermatol Surg. 2020;24(1):1-4. https://doi.org/10.4103/jdds.jdds_54_19

13. Al-Imam A. Optimizing linear models via sinusoidal transformation for boosted machine learning in medicine. J Facult Med Baghdad. 2019;61(3-4):128-136. https://doi.org/10.32007/jfacmedbagdad.613,41713

14. Al-Imam A. A novel method for computationally efficacious linear and polynomial regression analytics of big data in medicine. Modern Appl Sci. 2020;14(5):1-10. https://doi.org/10.5539/mas.v14n5p1

15. Al-Imam A, Abdul-Wahaab IT, Konuri VK, Sahai A, Al-Shalchy AK. Unification of Frequentist Inference and Machine Learning for Pterygomaxillary Morphometrics. Folia Morphologica. 2021; 80(3): 625-641. https://doi.org/10.5603/FM.a2020.0149

16. Al-Imam A. Inferential analysis of big data in real-time: One giant leap for spatiotemporal digital epidemiology in dentistry. Odontostomatol Res Anat Learn Implantol. 2019;12(1):1-14.

17. Oxford Centre for Evidence-Based Medicine. Oxford Centre for Evidence-Based Medicine: Levels of Evidence; 2009. Available from: https://www.cebm.ox.ac.uk/resources/levels-of-evidence/ oxford-centre-for-evidence-based-medicine-levels-of-evidencemarch-2009 [Last accessed on 2021 Apr 29].

\section{Authors Contribution:}

HJJ, SHA - Developed the study concept, reviewed the literature, and wrote the first draft of the manuscript; AA - Conceived the aims of the study, retrieved the raw data, conducted data analytics, and wrote the first draft of the manuscript; HAH - Wrote the case report; AKA - Revised the final draft of the article.

\section{Work attributed to:}

College of Medicine, University of Baghdad, Iraq.

Orcid ID:

Ahmed Al-Imam - (1) https://orcid.org/0000-0003-1846-9424

Source of Support: Nil, Conflicts of Interest: None. 